\title{
Optimization of Energy in Wireless Sensor Networks by Connectivity and Scheduling of Sensor Activity
}

\author{
Abdoulaye Ali Ibrahima ${ }^{1}$, Saley Bisso ${ }^{1,2}$, Salimata Gueye Diagne $^{1} \&$ Dthi Dione $^{2}$ \\ ${ }^{1}$ Cheikh Anta Diop University of Dakar Senegal \\ ${ }^{2}$ Abdou Moumouni University of Niamey (Niger) \\ Correspondence: Abdoulaye Ali Ibrahima, Cheikh Anta Diop University of Dakar Senegal. \\ E-mail: ibrahima.a.ali@aims-senegal.org
}

Received: October 9, 2020 Accepted: November 26, 2020 Online Published: Novmeber 30, 2020

doi:10.5539/jmr.v12n6p116 URL: https://doi.org/10.5539/jmr.v12n6p116

\begin{abstract}
In this article, we have addressed issues related to the optimization of the coverage and connectivity of a wireless sensor network by scheduling sensor activity with coverage and connectivity criteria. We presented a linear formulation of the coverage and connectivity that we solve with CPLEX. The results obtained show that sensor scheduling can improve the lifetime of a network by maintaining its coverage and connectivity.
\end{abstract}

Keywords: optimization, coverage, connectivity, activity scheduling, sensor network

\section{Introduction}

Recent technological developments in electronics, mechanics and also in the field of communication have given rise to a new generation of networks, the wireless sensor networks. This new era of embedded computing promises to revolutionize the way we live, work and interact with the physical environment around us. Sensor nodes that communicate via wireless links and that have computing and data collection capabilities facilitate a wide range of applications that were impractical or overpriced a few years ago.the sensors are powered by batteries with limited power, when the sensor node runs out of power it will be considered dead. In addition, recharging batteries in sensor networks is sometimes impossible due to the inaccessible location of the nodes or simply because it is practically or economically unfeasible. They must therefore be operated in an energy-efficient manner. As a result, the implementation of good energy management policies remains a much debated issue among researchers. The objective of this paper is to propose a mathematical model that minimizes the number of active sensors under the constraints that the whole area is covered and the total connectivity of the network is ensured. Wireless sensor networks are intended to collect information in hostile environments to which humans do not always have access. This is why it is considered that once they are deployed, the sensors are autonomous. Their lifespan is therefore the lifespan of their battery.

In sensor networks, the constraints imposed on communication protocols depend on the nature of the sensors as the application made of them. However, the energy constraint is considered as a stronger constraint, since we consider that the sensors are generally deployed in hostile areas of interest and therefore the recharging their batteries is almost impossible. In addition, the Related Dominant Set (CDS) approach was used to optimize communication costs. (J.Carle \& D. Simplot-Ryl.,2004), the reduction of the diffusion overhead consists of reduce the size and cost of maintaining routing tables by removing all links between nodes that are not part of the backbone formed by the connected dominant set, and involving only the nodes forming the backbone in the diffusion. Thus, excessive redundancy emission could be avoided. (B. Chen et al.,2002), the performance of the diffusion is optimized thanks to the involvement only of the nodes forming the backbone in maintaining the connectivity of the network and putting the other nodes in sleep mode. Furthermore, (G.Hoblos et al.,2000), implied the associated dominant set technique to provide both area coverage and routing information from sensors to the base station in sensor networks deployed in large numbers. Some sensors could be faulty, blocked due to the depletion of their batteries, or suffer physical damage (run over by animals or when thrown by an airplane).The failure of these sensors should not have an influence on the operation of the network. Fault tolerance is the ability to support the functionality of a sensor network without causing disruption when a sensor stops functioning Most of the solutions offered for area coverage in networks of sensors are based on a flat view where all the nodes are considered equal and must together contribute to the management of the network so that they can accomplish its tasks. Through elsewhere, the sensors can appear, disappear (by switching off their radio, exhaustion of their energy or crash), or move independently of each other. Thus, the topology of the network is constantly evolving. As a result, the routing of information from a sensor to a remote base station and vice versa must be done by an energy efficient protocol. In order for this protocol to be effective, it must take into account the intrinsic constraints network (constantly evolving topology), the 
limited sensor resources in terms of One of the commonly proposed solutions for broadcast and area coverage in wireless sensor networks is to use a hierarchical architecture called still clustering. This architecture makes it possible to group geographically close sensors into clusters and use different routing schemes within and between clusters. We present in detail the activity scheduling with the criteria of coverage and connectivity. We will first present a coverage model and then its improvement with the addition of connectivity constraints. The presentation of these models will be preceded by their descriptions followed by the terminologies used in them. The last part of this article will be devoted to numerical tests followed by discussion. Then a conclusion with some perspectives.

\section{Generality}

Recent technological advances have reinforced the presence of computers and electronics at the heart of the real world. More and more objects are being equipped with processors and mobile communication devices, enabling them to process information and also to transmit it. Wireless sensor networks fall within this framework. The main purpose of a wireless sensor network is to capture information, process it if necessary and then send it to the base station, which then transmits it via the Internet to an end user. Thus, complete coverage of the area of interest as well as full network connectivity is fundamental to any wireless sensor network. We propose a linear formulation of the connectivity to guarantee the acquisition of the collected information while ensuring the coverage of the area to detect all the targets that will be present there. A sensor node is composed of several elements or modules, each corresponding to a particular task of data acquisition, processing, or transmission. A wireless sensor network is composed of two types of nodes: sensor nodes and well nodes. A wireless sensor network is composed of two types of nodes: sensor nodes and sink nodes. The sensors are responsible for collecting and processing information and routing the information collected over the coverage area to the collection point, also known as a sink. The well node retrieves the information collected by the various sensors and transmits it to the processing centre. The randomly arranged sensors form the coverage area. Some sensors could be faulty, blocked due to depletion of their batteries, or suffer physical damage (run over by animals or when thrown by an airplane). The failure of these sensors should not have an influence on the operation of the network. Fault tolerance is the ability to support the functionality of a sensor network without causing disruption when a sensor stops functioning(G.Hoblos et al.,2000) .However, when the number of nodes increases(B. Chen et al.,2002) or the nodes are mobile(Antoine Gallais.,2007), the control traffic predominates the communications real. Which leads to an increase in latency and an explosion of routing. Since the sensor node is the core component to form wireless sensor network, a general hardware architecture of it is presented in Fig.1 The architecture can be divided into four parts: processing unit, communication unit, sensing unit and power supply unit. Usually, the sensing unit is made up of various kinds of sensors to measure a physical quantity and convert it into an electrical signal that can be read by the processing unit. The analogue signals produced by the sensing unit are converted to digital signals by an analogue to digital converter (ADC) in the processing unit, and further data processing can be done if necessary (e.g., data encoding, data compression). Besides, the processing unit is also responsible for the task management of the node. After that, the communication unit enables wireless communication between different nodes. The power supply unit provides the supply voltage for the whole sensor node to guarantee the running of all on-node components. Currently, many commercial-off-the-shelf (COTS) sensor nodes offer feasible options. These nodes have already been widely used by many academic institutions and industrial departments. Some well-known and typical nodes are the MICA mote and Telos mote series. MICA2 was designed by UC Berkeley and released by Crossbow Tech Inc. in 2002. The MICA2 node can be used for several different sensors (e.g., temperature, light, magnetisme, acoustics, etc), and Atmel ATmega128L MCU(Atmel corporation.,2011) is used to connect sensors and transceivers. The CC1000 transceiver (Texas Intruments.,2009) supports the maximum data rate of $76.8 \mathrm{Kbps}$ and the default data rate of MICA2 is selected as $38.4 \mathrm{Kbps}$. As an improved version of MICA2, MICAz replaces the CC1000 radio with CC2420 (CC2420 datasheet.,2013) which is an IEEE 802.15.4 (IEEE Computer Society.2006) compatible transceiver and supports the maximum data rate of $250 \mathrm{Kbps}$. Compared with the MICA series, Telos (Moteiv Corporation.,2006) is a newly developed mote released in 2004. It still uses CC2420 radio, but replaces ATmega128L MCU with a very low-power MSP430 MCU. Other motes that share the same structure of Telos include TelosB , Tmote Sky (Moteiv Corporation.,2006) . In addition to these low-power based platforms, some high-power based platforms with more powerful MCUs can be used for data processing and data routing tasks in wireless sensor network. Examples $\mu$ AMPS (Rex Min et al.,2002).

A wireless sensor network is made up of two types of nodes: sensor nodes and sink nodes. The sensors are responsible for collecting information, processing it if necessary and routing the information collected over the coverage area to the collection point, also called sink. The sink node retrieves the information sent by the various sensors and transmits them at the treatment center. The randomly arranged sensors form the coverage area. The figure below is an illustration of a wireless sensor network. 


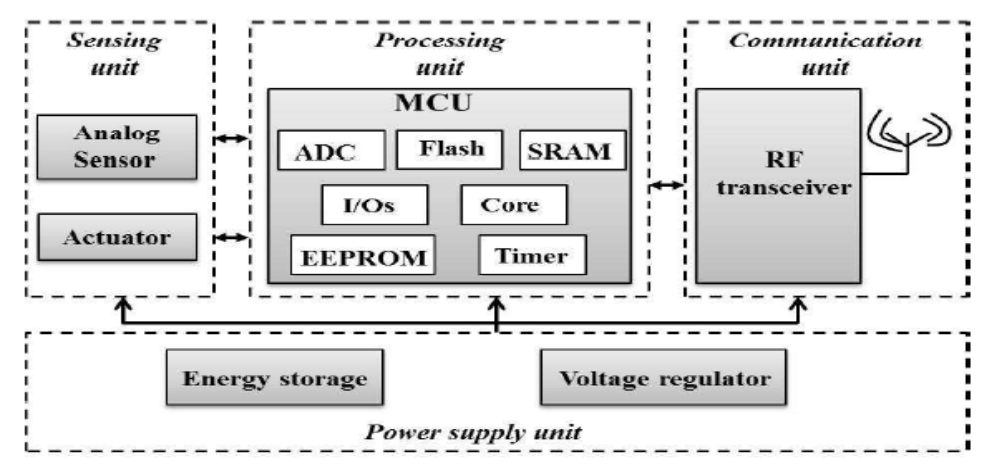

Figure 1. Structure of a sensor node.

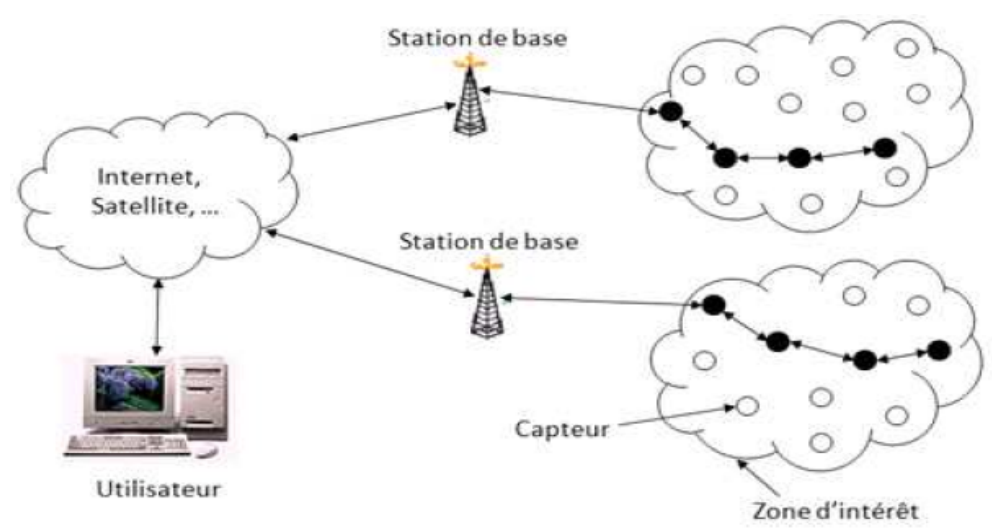

Figure 2. Architecture of wireless sensor network

\subsection{Fields of Application of Sensor Networks}

The evolution of wireless communication media, miniaturization, the low cost of micro-sensors and the widening range of sensor probes have broadened the field of application of sensor networks. They allow complex information from the environment etc. to be collected and processed. They can also be used in other industrial systems such as the control and automation of disassembly lines. Sensor networks could also revolutionize the very way complex physical systems are understood and built, particularly in the military, environmental, domestic, health, security and other fields. Researchers at the Lawrence Livermore National Laboratory have set up the WATS network (Wide Area Tracking System). This network is made up of detectors gamma rays and neutrons to detect and track nuclear devices. It is capable of constantly monitoring an area of interest.

\subsection{Energy Optimization Approach in Sensor Networks}

One of the major advantages of wireless sensor networks is the ability to monitor targets over a wide area at a lower cost. The sensors are autonomous and are usually powered by a battery with a limited lifetime. Therefore, energy consumption is an important aspect of monitoring missions. In general, it is not useful to activate all the sensors at the same time to monitor all the targets (Fellah Kahadidja.,2008), as many parts of the area to be monitored are covered by several sensors at the same time. Maximizing the life of the network means reducing the energy consumption of the nodes. Despite the progress that has been made, the lifetime of these battery-powered devices continues to be a major challenge and a key factor, requiring more research into the energy efficiency of communication platforms and protocols. Assuming that targets and their trajectories are known in advance, our goal is to plan a schedule of sensor activities. This scheduling should be such that it preserves, as much as possible, the current ability of the sensor network to subsequently monitor certain areas of interest to the sensor network operator. It is also desired that the scheduling minimizes the total energy consumed during the monitoring mission(Rahim Kacimi.,2009). The context of sensor network use is now one of multiple missions, where the primary goal is to preserve the network for future monitoring missions, while at the same 
time focusing on solutions that minimize the energy consumed to accomplish the current mission. Concept of network life The goal of any designer is to implement a network that lasts as long as possible. The lifetime of a network is defined as the time that has elapsed since its deployment until the network is non-functional. The term non-functional depends on the type of application being considered, so the lifetime is strongly related to the expected results. Thus we can have a lifetime based on coverage and a lifetime based on connectivity. Recurring problem of the lifetime of a sensor network In the different routing protocols we almost always find the same problem that recurs all the time. It is the life span of the network that is still limited. Indeed, by trying to change the routing protocol, we can obtain energy savings but the network may no longer function because nodes whose position in the network is considered strategic have exhausted their energy.

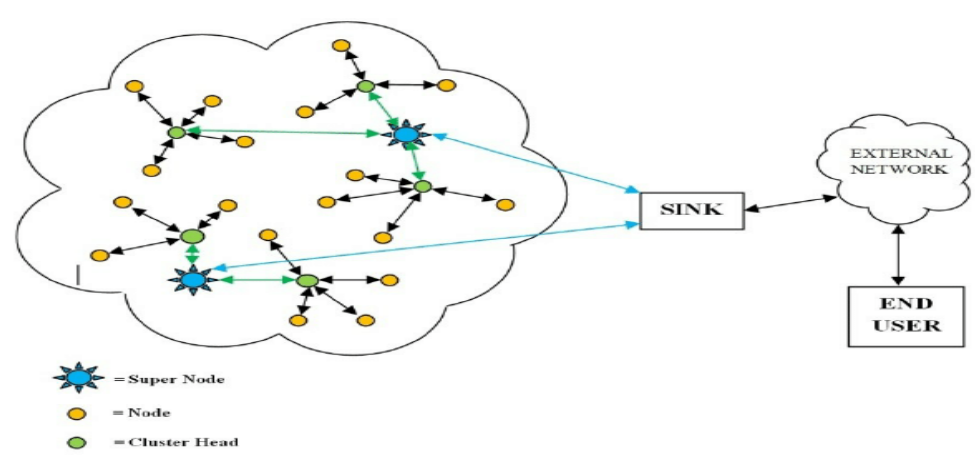

Figure 3. Critical nodes in a network

\subsection{Sensor Scheduling in the RCSFs}

Wireless networks are essentially made up of sensor nodes, the duration of which depends on the life of their batteries. Scheduling of node activity is one of the techniques used to improve sensor energy consumption. Scheduling is the selection of nodes deployed in the network to remain active while maintaining the essential purpose of the network. Therefore, regardless of the state of the sensors, the area of interest must be covered as completely as possible; each active node set must form a connected network so that it can carry information to the base station. The scheduling problem can be summarized as follows: The goal is to minimize the number of active nodes while maintaining the primary goal of the network, which is complete coverage of the study area and total network connectivity. Scheduling is a technique that manages the redundancy of nodes during deployment in order to save sensor energy (Dame Diongue.,2014;Rahim Kacimi.,2009). It involves putting redundant sensors on standby while preserving the coverage of the area of interest and maintaining network connectivity(Pascale Minet et al.,2013). Therefore, to design a scheduling mechanism that meets the application requirements, the following questions must be answered:

1. What is the rule to follow so that a node knows whether to enter standby mode?

2. 2. When should a node make such a decision?

3. How long should a node remain in standby mode?

\section{Distributed Scheduling Algorithm in CWHNs}

In this section we present a distributed scheduling algorithm. It allows the sensor nodes to make a decision whether to be in active or standby mode. The algorithm is composed of two phases:

-a self-scheduling phase and a monitoring phase. Self-scheduling phase The self-scheduling phase consists of two parts:

1. Each node sends its neighbours a message containing its location, energy reserve, identity and degree. The message is called MDC (Message D Sensors). On receipt of MDC, each node compares itself to the sender and maintains two lists of neighbours, a list called LCS (List of Upper Sensors) and a list called LCI (List of Lower Sensors) depending on the type of message received. The LCS list is the list intended for storing the sensors dominating the sender and the LCI list is intended for storing the sensors dominated by the sender. 
2. At the end of the previous step, each sensor checks if its perception area is covered by the nodes of its LCS list.

If yes, then it deactivates itself directly. If not, it waits for a certain period of time until it is connected to its energy reserve and then recalculates its eligibility for switch-off by considering: LCS nodes LCI nodes have already decided to be active. If it is eligible then it will deactivate. Otherwise it sends an awake message to its neighbors and restores itself. -Monitoring phase Monitoring phase After the self-scheduling phase, the active nodes monitor the study area. $\bullet$ Example To illustrate the algorithm, let's consider the figure below. It is assumed that nodes $2,3,5,6$, have higher energy reserves than nodes 1 and 4 . So two cases occur between node 1 and node 4:

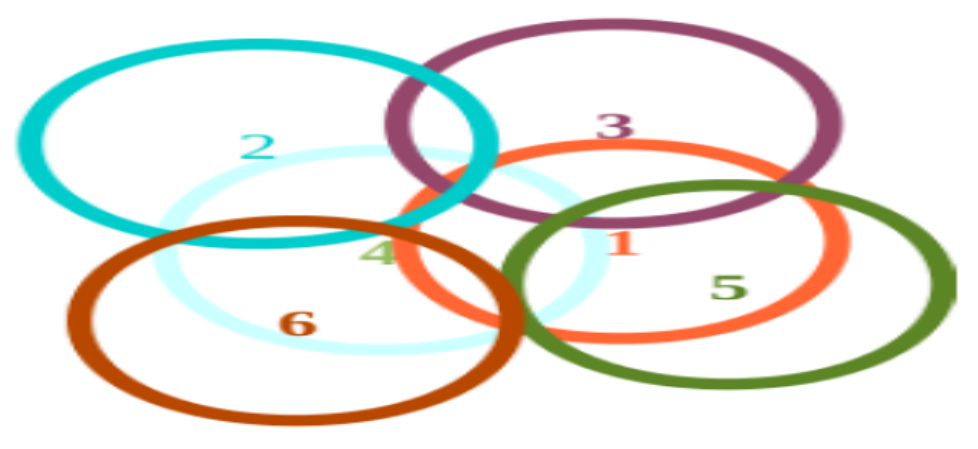

Figure 4. Examples

1. The energy of node 1 is less than the energy of node 4 .

2. The energy of Node 4 is less than the energy of Node 1.

For the first case we have : $\bullet \operatorname{LCS}(1)=\{3,4,5\} \Longrightarrow 1$ is eligible. $\bullet \operatorname{LCS}(4)=\{2,6\} \Longrightarrow 4$ must be active. For the first case we have: $\bullet \operatorname{LCS}(1)=\{3,4,\} \Longrightarrow 1$ must remain active. $\bullet \operatorname{LCS}(4)=\{1,2,6\} \Longrightarrow 4$ must remain active.

\section{Modeling the Problem}

\subsection{Description and Ratings}

In this section we propose a model that manages the redundancy resulting from the deployment in the sensor networks to maximize the life of the network while guaranteeing the total coverage of the area of interest as well as the network connectivity. The goal is to minimize the number of active nodes in order to save standby power when constrained by application requirements. To do so, we consider an area of interest containing more nodes than necessary for its observation. The proposed model is based on the following assumptions: The study area is a grid. Deployment is random and redundant. The nodes are static. The nodes have the same coverage and communication radii. The nodes cannot be reloaded. (LEHSAINI Mohamed.,2009) are detailed the existing works The terminologies used in the model are summarized in the table below

Table 1. Notations used in the model

\begin{tabular}{ll}
\hline NOTATION & Meaning \\
\hline $\mathrm{M}$ & All the positions of the grid sensors \\
\hline $\mathrm{N}$ & All the positions of the grid targets \\
\hline $\mathrm{Ti}$ & The number of intermediate sensors between the sensor present at position $\mathrm{i}$ and the base station \\
\hline $\mathrm{d}(\mathrm{i}, \mathrm{j})$ & Distance between the sensor at position $\mathrm{i}$ and the target at position $\mathrm{j}$ \\
\hline $\mathrm{Rcom}$ & Communication radius \\
\hline $\mathrm{Rcov}$ & Coverage radius \\
\hline $\mathrm{Si}$ & Binary value equal to 1 if the distance between the sensor at position $\mathrm{i}$ and the base station is \\
less than or equal to Rcom and 0 otherwise & \\
\hline aij & Binary value equal to 1 if $\left.d_{(} i ; j\right) \leq R \operatorname{Rov}$ and 0 otherwise \\
\hline bik & Binary values is equal to 1 if $\left.d_{(} i ; k\right) \leq R \operatorname{Rom}$ and 0 otherwise \\
\hline
\end{tabular}




\subsection{Mathematical Formulation}

In this section we first give a model that ensures the coverage of the area of interest and then a model that guarantees both the coverage of the study area and the connectivity of the network.

1. Model under coverage constraint :

-The decision variable :

The decision variable is the binary value $\mathrm{Xi}$ which expresses the state of the sensor at position i. It is defined by : $X_{i}=\left\{\begin{array}{ll}1 & \text { if the sensor at position i is active } \\ 0 & \text { otherwise }\end{array}\right.$ The objective function : The objective is to minimize the number of active nodes present in the area of interest. The objective function is given by :

$$
Z=\sum_{i \in M} X_{i}
$$

-Constraints :

In order to ensure full coverage of the area, each position in the zone must be monitored by at least one sensor. So the coverage constraints are :

$$
Z=\sum_{i \in M} a_{i, j} X_{i} \quad \forall j \in N
$$

- The model :

Thus the problem of minimizing the number of active sensors under stress is written :

$$
\begin{gathered}
\min \sum_{i \in M} X_{i} \\
\sum_{i \in M} a_{i, j} X_{i} \geq 1 \quad \forall j \in N \\
X_{i} \in\{0,1\} \quad \forall i \in M
\end{gathered}
$$

1. Modle Model under coverage and connectivity constraints :

Covering an area is useless if it is not possible to transmit the collected information to the base station. Therefore, connectivity is a fundamental constraint for the proper functioning of any network. Thus, we propose a model that minimizes active nodes and ensures both area coverage and network connectivity.

-The decision variable :

To ensure connectivity within the model, we choose to add to the decision variable an index t characterizing the degree of connectivity. It thus becomes $X_{i, t}$ and is defined :

$X_{i, t}=\left\{\begin{array}{ll}1 & \text { if sensor } \mathrm{i} \text { is active and is connected to the base station via } \mathrm{t} \text { active } \\ 0 & \text { otherwise }\end{array}\right.$ In other words, there is a path between position $\mathrm{i}$ and the main station consisting of $\mathrm{t}$ sensors, each successive pair being at a distance less than or equal to $R_{\text {com }}$. -The objective function :

The objective is to minimize the number of active nodes present on the grid in order to maximize the life of the network. Thus the function to be minimized is

$$
Z=\sum_{i \in M} \sum_{t=0}^{T_{i}} X_{i, t}
$$

- Contraintes de couverture :

As we added a t dimension to the decision variables, the hedging constraint is written :

$$
Z=\sum_{i \in M} \sum_{t=0}^{T_{i}} a_{i, j} X_{i, t} \geq 1 \quad \forall j \in N
$$


-Connectivity constraint :

It is divided into two parts: direct connection and indirect connection Direct :it corresponds to the grid elements that are in direct range of the base station, i.e. case $\mathrm{t}=0$. It is ensured by the constraint:

$$
S_{i}-X_{i, 0} \geq 0 \quad \forall i \in M
$$

Indirect connection : it corresponds to the grid elements linked by the base station by at least one intermediate sensor, i.e. case $\mathrm{t} i 0$. The indirect connection is ensured by the following constraint :

$$
\sum_{k \in M} b_{i, k} X_{k, t-1}-X_{i, t} \geq 0 \quad \forall i \in M \text { and } t=1, \ldots . ., T_{i}
$$

-The model :

The global linear model that expresses coverage and connectivity is written as follows :

$$
\begin{array}{r}
\min \sum_{i \in M} \sum_{t=0}^{T_{i}} X_{i, t} \\
\sum_{i \in M} \sum_{t=0}^{T_{i}} a_{i, j} X_{i, t} \geq 1 \quad \forall j \in N \\
\sum_{k \in M} b_{i, k} X_{k, t-1}-X_{i, t} \geq 0 \quad \forall i \in M \quad \text { et } \quad t=1, \ldots . ., T_{i} \\
S_{i}-X_{i, 0} \geq 0 \quad \forall i \in M \\
X_{i, t} \in\{0,1\} \quad \forall i \in M \quad \text { et } t=1, \ldots . ., T_{i}
\end{array}
$$

The proposed model thus involves minimizing the number of active sensors ( 6 ) knowing that each target in the area must be covered by at least one sensor (7). Each sensor is connected directly to the base station (9) or is linked to the base station by $t$ active sensors (8).

\section{Digital Tests}

The digital tests were carried out with the CPLEX studio software. It allows to create files according to the OPL language. The OPL language is a computer language allowing to specify optimization problems, which can then be passed to a solver for resolution. These files allow to express continuous linear optimization problems, integer linear optimization problems or mixed constraint programming problems. However, it does not allow the expression of non-linear programming problems.

\section{Cover Model}

-Parameters used

For the coverage model we consider an area of interest containing 10 sensors for the monitoring of 7 targets. Its coverage matrix is represented by a table with $c_{j} ; \mathrm{j}=1 ; \ldots ; 7$ represent the targets. with $x_{i}$ the number of sensors; $\mathrm{i}=1 ; \ldots ; 10$.

Table 2. Cover matrix

\begin{tabular}{|c|c|c|c|c|c|c|c|}
\hline & $C_{1}$ & $C_{2}$ & $C_{3}$ & $C_{4}$ & $C_{5}$ & $C_{6}$ & $C_{7}$ \\
\hline$x_{1}$ & 1 & 0 & 1 & 0 & 0 & 0 & 0 \\
\hline$x_{2}$ & 0 & 1 & 0 & 1 & 0 & 0 & 0 \\
\hline$x_{3}$ & 0 & 0 & 0 & 0 & 0 & 0 & 1 \\
\hline$x_{4}$ & 1 & 1 & 0 & 0 & 0 & 1 & 0 \\
\hline$x_{5}$ & 0 & 0 & 1 & 0 & 1 & 1 & 1 \\
\hline$x_{6}$ & 0 & 0 & 1 & 0 & 0 & 0 & 0 \\
\hline$x_{7}$ & 1 & 0 & 1 & 1 & 0 & 0 & 0 \\
\hline$x_{8}$ & 0 & 1 & 0 & 0 & 0 & 1 & 0 \\
\hline$x_{9}$ & 0 & 0 & 0 & 0 & 0 & 0 & 1 \\
\hline$x_{10}$ & 0 & 1 & 0 & 0 & 1 & 1 & 0 \\
\hline
\end{tabular}


- Results and analysis

The solution of the problem with CPLEX, whose solution program is shown in the appendix, gave the following solutions: $X_{1}=0 ; X_{2}=0 ; X_{3}=0 ; X_{4}=1 ; X_{5}=1 ; X_{6}=0 ; X_{7}=1 ; X_{8}=0 ; X_{9}=0 ; X_{10}=0$

Thus only the activation of 3 sensors is sufficient for the observation of all the targets present in the area. The other sensors will therefore be in standby mode and will save their energy, which will result in a longer network life while maintaining full coverage of the area.

\section{Coverage and Connectivity Model}

\section{-Parameters Used}

For the coverage and connectivity model we considered an area containing 7 randomly deployed sensors with the maximum number of sensors fixed at 2 whose coverage and connectivity matrixes are represented respectively in Tables 3 and 4 and the $S_{i}$ vector is given by the table 5 .

Table 3. Cover matrix

\begin{tabular}{|l|l|l|l|l|l|l|}
\hline 1 & 0 & 1 & 0 & 0 & 0 & 0 \\
\hline 0 & 1 & 0 & 1 & 0 & 0 & 0 \\
\hline 0 & 0 & 0 & 0 & 0 & 0 & 1 \\
\hline 1 & 1 & 0 & 0 & 0 & 1 & 0 \\
\hline 0 & 0 & 1 & 0 & 1 & 1 & 1 \\
\hline 0 & 0 & 1 & 0 & 0 & 0 & 0 \\
\hline 0 & 1 & 0 & 0 & 1 & 1 & 0 \\
\hline
\end{tabular}

Table 4. Connectivity matrix

\begin{tabular}{|l|l|l|l|l|l|l|}
\hline 1 & 0 & 1 & 0 & 0 & 0 & 0 \\
\hline 0 & 1 & 1 & 1 & 0 & 0 & 0 \\
\hline 0 & 0 & 0 & 0 & 0 & 0 & 1 \\
\hline 1 & 1 & 1 & 0 & 0 & 1 & 0 \\
\hline 0 & 0 & 1 & 0 & 1 & 1 & 1 \\
\hline 0 & 1 & 1 & 0 & 0 & 0 & 0 \\
\hline 1 & 0 & 1 & 1 & 1 & 0 & 0 \\
\hline
\end{tabular}

Table 5. The vector $S_{i}$

\begin{tabular}{|l|l|l|l|l|l|l|}
\hline 1 & 0 & 1 & 0 & 0 & 0 & 1 \\
\hline
\end{tabular}

-Results and Analysis

The resolution of the model with CPLEX whose resolution program gave the following results: : 
Table 6. Results

\begin{tabular}{|c|c|c|}
\hline Mm (Value) & Tt (degree) & Solution \\
\hline 1 & 0 & 0 \\
\hline 1 & 1 & 0 \\
\hline 1 & 2 & 0 \\
\hline 2 & 0 & 0 \\
\hline 2 & 1 & 0 \\
\hline 2 & 2 & 1 \\
\hline 3 & 0 & 0 \\
\hline 3 & 1 & 0 \\
\hline 3 & 2 & 0 \\
\hline 4 & 0 & 0 \\
\hline 4 & 1 & 0 \\
\hline 4 & 2 & 0 \\
\hline 5 & 0 & 0 \\
\hline 5 & 1 & 0 \\
\hline 5 & 2 & 0 \\
\hline 6 & 0 & 0 \\
\hline 6 & 1 & 0 \\
\hline 6 & 2 & 0 \\
\hline 7 & 0 & 0 \\
\hline 7 & 1 & 0 \\
\hline 7 & 2 & 0 \\
\hline
\end{tabular}

Thus the activation of two sensors is sufficient to ensure the coverage and connectivity of the network. Thus the activation of two sensors is sufficient to ensure the coverage and the connectivity of the network. These are the $\mathrm{x} 2$ and $\mathrm{x} 3$ sensors of respective degrees 1 each Thus the information captured by $\mathrm{x} 2$ passes through $\mathrm{x} 3$ to reach the base station and the information captured by $\mathrm{x} 3$ passes through $\mathrm{x} 2$ to reach the base station. The sensor $\mathrm{x} 1$ will be in standby mode and therefore will save its energy. Hence the increase of the network life time.

\section{Conclusion}

In this article we have dealt with the optimization of coverage and connectivity of wireless sensor networks. First we presented a general overview of sensor networks. We also studied the scheduling of sensor activities. It is a technique used to overcome the various constraints mentioned above, particularly the energy constraint. It involves activating the necessary number of nodes and putting the others on standby. The nodes in standby mode will save their energy so the life of the network will be extended. We have proposed a mathematical model whose goal is to minimize the number of active nodes under constraint of the application requirements, i.e. the total coverage of the area of interest as well as the network connectivity. And finally the resolution of the model obtained with the Ilog CPLEX solver which gave solutions that show that the distributed scheduling of the nodes allows us not only to consume less energy but also to reduce the complexity of the network. The work of this paper has been carried out under the following assumptions :

1. The nodes deployed are static

2. The nodes have the same coverage and connectivity radius

3. The base station is unique

For future work, we plan to expand our work with a heterogeneous CWHN - a network with mobile nodes and a diversity of communication radius and coverage. The proposed model considers an area of interest with a single base station. An interesting perspective of this work would also be to propose a model with several base stations.

\section{References}

Abdallah, M. (2008). Sensor networks: location, coverage and data fusion. Thesis of the University France counts.

Antoine, G. (2007). Scheduling of Activity in Sensor Networks: the Example of Surface Coverage. Thesis in Mathematical Sciences from the University of Sciences and Technologies of Lille (computer science specialty) presented on June 26. https://doi.org/10.1002/nba.30737 
Atmel Corporation. (2011). "Atmega128 datasheet," [Online]. Retrieved from http://www.atmel.com/Images/doc2467.pdf (Cited on pages 7 and 46.)

BENAHMED, K. (2007). Graph Theory Approach for Monitoring a Wireless Sensor Network. For obtaining the degree of Magister in Computer Science from the University of Oran Es-senia.

Carle, J., \& Simplot-Ryl, D. (2004). Energy-efficient area monitoring for sensor networks. Computer, 37(2), 40-46. https://doi.org/10.1109/MC.2004.1266294

Cc2420 datasheet. (2013). [Online]. Retrieved from http://www.ti.com/lit/ds/symlink/cc2420.pdf (Cited on pages 7, 13, 40 and 62.) Cheick-Tidjane, K. (2011). Design of the architecture of a large-scale wireless sensor network. Doctor of Henri Poincare University, Nancy I.

Chen, B., Jamieson, K., Balakrishnan, H., \& Morris, R. (2002). Span: An energy-efficient coordination algorithm for topology maintenance in ad hoc wireless networks. Wireless networks, 8(5), 481-494. https://doi.org/10.1145/381677.381686

Chrif, D. (2010). Routing and training improvement techniques multi-hop clusters in sensor networks wireless.

Dame Diongue. (2014). Distributed lifetime optimization in wireless sensor networks. Doctoral thesis from the University of Gaston Berger de Saint Louis Specialty IT supported on June 13.

Du, W. (2011). Modeling and simulation of wireless sensor networks (Doctoral dissertation).

Fellah, K. (2008). OPTIMIZATION TECHNIQUES FOR THE ECONOMY ENERGY IN WIRELESS SENSOR NETWORKS. Master's degree in Computer Science from the University of Oran Es-senia.

Gosnell, T. B., Hall, J. M., Jam, C. L., Knapp, D. A., Koenig, Z. M., Luke, S. J., ... Wolford, J. K. (1997). Gamma-ray identification of nuclear weapon materials (No. UCRL-ID-127436). Lawrence Livermore National Lab., Livermore, CA (US).

Hoblos, G., Staroswiecki, M., \& Aitouche, A. (2000, September). Optimal design of fault tolerant sensor networks. In Proceedings of the 2000. IEEE International Conference on Control Applications. Conference Proceedings (Cat. No. OOCH37162) (pp. 467-472). IEEE.

IEEE Computer Society. (2006). Part 15.4. Wireless medium access control (mac) and physical layer (phy) specifications for low-rate wireless personal area networks (wpans). IEEE Std 802.15.4-2006. (Cited on pages 3, 7, 19, 20 and 46.)

Tian, D., \& Georganas, N. D. (2002, September). A coverage-preserving node scheduling scheme for large wireless sensor networks. In Proceedings of the 1st ACM international workshop on Wireless sensor networks and applications (pp. 32-41). https://doi.org/10.1145/570738.570744

Rahim KACIMI. (2009). Energy conservation techniques for wireless sensor networks. Doctorate from the University of Toulouse.

LEHSAINI, M. (2009). Diffusion and coverage based on clustering in sensor networks: application to home automation. PhD thesis of the University of Franche-Comt, Computer Science, year .

Min, R., Bhardwaj, M., Cho, S. H., Ickes, N., Shih, E., Sinha, A., ... Chandrakasan, A. (2002). Energy-centric enabling technologies for wireless sensor networks. IEEE Wireless Communication Magazine, 9(4), 28-39.

Moteiv Corporation. (2004). "Telos datasheet," [Online].

Retrieved from http://www.eecs.harvard.edu/ konrad/References/TinyOSDocs/telos-reva-datasheet-r.pdf (Cited on pages 7 and 40.)

Moteiv Corporation. (2006). "Tmote sky datasheet." [Online].

Retrieved from http://www.eecs.harvard.edu/ konrad/projects/shimmer/references/tmote-sky-datasheet.pdf (Cited on pages 3, 7, 9, 40 and 46.)

Pascale, M., Ridha, S., Ichrak, A., Erwan, L., \& Saoucene, M. (2013). Scheduling of node activity in ad hoc networks and wireless sensor networks. Real Time Summer School, Aug, Toulouse, France.

Quentin, L. (2014). Urban networks of wireless sensors: Applications, characterization and protocols. Thesis in Computer Science The National Institute of Applied Sciences of Lyon, January.

Said El Abdellaoui. (2013). Study and analysis of the performance of the cooperative "MIMO-Virtual" system for wireless sensor networks. MOHAMMED V-AGDAL Rabat Science Thesis, year. 
Texas Instruments, Inc, ”Cc1000 datasheet”, [Online]. Retrieved from http://www.ti.com/lit/ds/symlink/cc1000.pdf, 2009. (Cited on pages 7 and 62.)

\section{Copyrights}

Copyright for this article is retained by the author(s), with first publication rights granted to the journal.

This is an open-access article distributed under the terms and conditions of the Creative Commons Attribution license (http://creativecommons.org/licenses/by/4.0/). 